\title{
Interacting endogenous and exogenous RNAi pathways in Caenorhabditis elegans
}

\author{
ROSALIND C. LEE, CHRISTOPHER M. HAMMELL, and VICTOR AMBROS
}

Dartmouth Medical School, Department of Genetics, Hanover, New Hampshire 03755, USA

\begin{abstract}
C. elegans contains numerous small RNAs of $\sim 21-24$ nt in length. The microRNAs (miRNAs) are small noncoding RNAs produced by DCR-1- and ALG-dependent processing of self-complementary hairpin transcripts. Endogenous small interfering RNAs (endosiRNAs), associated with ongoing silencing of protein-coding genes in normal worms, are produced by mechanisms that involve DCR-1 but, unlike miRNAs, also involve RDE-2, RDE-3, RDE-4, RRF-1, and RRF-3. The tiny noncoding (tncRNAs) are similar to endo-siRNAs in their biogenesis except that they are derived from noncoding sequences. These endo-siRNA- and tncRNA-based endogenous RNAi pathways involve some components, including DCR-1 and RDE-4, that are shared with exogenous RNAi, and some components, including RRF-3 and ERI-1, that are specific to endogenous RNAi. rrf-3 and eri-1 mutants are enhanced for some silencing processes and defective for others, suggesting cross-regulatory interactions between RNAi pathways in $C$. elegans. Microarray expression profiling of RNAi-defective mutant worms further suggests diverse endogenous RNAi pathways for silencing different sets of genes.
\end{abstract}

Keywords: noncoding RNA; siRNA; microRNA; RNAi; RdRp; Caenorhabditis elegans

\section{INTRODUCTION}

Evolutionarily conserved mechanisms for gene silencing mediated by small RNAs of 21-24 nt in length are found in essentially all eukaryotes (Ambros 2004; Baulcombe 2004; Lippman and Martienssen 2004; Mello and Conte 2004). One class of small RNAs is the microRNAs, which function in the regulation of development and physiology in multicellular eukaryotes. Another major class of small RNAs is the siRNAs, which function in a variety of transcriptional and post-transcriptional gene-silencing processes that are collectively referred to as RNA-mediated interference (RNAi). MicroRNAs and siRNAs are produced by the type III endonuclease Dicer from various forms of precursor doublestranded RNA (dsRNA), including short fold-back hairpin gene products (in the case of miRNAs), or, in the case of siRNAs, bidirectional transcripts, replicative intermediates from RNA-dependent RNA polymerase (RdRp) activity or possibly longer single-stranded transcripts with extensive secondary structure. RNAi can be elicited exogenously, by dsRNA supplied from outside the cell, or endogenously, from transcription of coding or noncoding genomic

Reprint requests to: Victor Ambros, Dartmouth Medical School, Department of Genetics, Hanover, NH 03755, USA; e-mail: vambros@ dartmouth.edu; fax: (603) 650-1188.

Article published ahead of print. Article and publication date are at http://www.rnajournal.org/cgi/doi/10.1261/rna.2231506. sequences (Ambros 2004; Baulcombe 2004; Lippman and Martienssen 2004; Mello and Conte 2004; Peragine et al. 2004; Allen et al. 2005; Grishok et al. 2005).

miRNAs and siRNAs function as guide RNAs, in conjunction with protein complexes containing members of the Argonaute family of RNA-binding proteins, to target complementary RNA transcripts for destruction or translational inhibition (Ambros 2004; Baulcombe 2004; Lippman and Martienssen 2004; Mello and Conte 2004). In some cases, including Caenorhabditis elegans, distinct Argonaute proteins function in conjunction with siRNAs and microRNAs, respectively (Tabara et al. 1999; Grishok et al. 2001; Tijsterman et al. 2002b; Vastenhouw et al. 2003). In other animals, the same Argonaute can function with both siRNAs and microRNAs (Williams and Rubin 2002; Meister et al. 2004; Okamura et al. 2004). Other proteins associated with the generation of siRNAs in C. elegans (Table 1) include RdRp, a nucleotidyltranferase $\beta$ family protein (RDE-3), and various helicases and RNA-binding proteins (Ketting et al. 1999; Tabara et al. 1999; Fagard et al. 2000; Smardon et al. 2000; Sijen et al. 2001; Morel et al. 2002; Simmer et al. 2002; Tabara et al. 2002; Tijsterman et al. 2002a; Tops et al. 2005).

Although RNAi has been shown to be involved in diverse processes such as the silencing of transposons, maintenance of heterochromatic DNA, and the defense against infection by RNA viruses (Ketting et al. 1999; Tabara et al. 1999; Mourrain et al. 2000; Morel et al. 2002; Sijen and Plasterk 2003; Grishok 
TABLE 1. Levels of representative small RNAs detected by Northern blot analysis of total RNA from strains defective in components of RNAi pathways

\begin{tabular}{|c|c|c|c|c|c|c|c|}
\hline \multirow[b]{2}{*}{ Genotype } & \multirow[b]{2}{*}{ Gene product ${ }^{a}$} & \multirow[b]{2}{*}{ RNAi function ${ }^{a}$} & \multicolumn{4}{|c|}{ Small RNA levels ${ }^{\mathrm{b}}$} & \multirow[b]{2}{*}{$\begin{array}{l}\text { Effects on endogenous } \\
\qquad R N A i^{f}\end{array}$} \\
\hline & & & $\operatorname{siRNA}{ }^{c}$ & $\operatorname{tncRNA}^{\mathrm{d}}$ & $\begin{array}{l}\text { miRNA } \\
\text { precursor }\end{array}$ & $\begin{array}{l}\text { mature } \\
\text { miRNA }^{\mathrm{e}}\end{array}$ & \\
\hline Wild type & & & 1.0 & 1.0 & 1.0 & 1.0 & \\
\hline $\begin{array}{l}\text { alg-1(RNAi); } \\
\quad \text { alg-2(ok304) }\end{array}$ & Argonaute & mir & 0.8 & 1.2 & 17 & 0.5 & microRNAs ${ }^{g}$ \\
\hline$d c r-1(o k 247)$ & Nuclease & mir, ex, co, tr & 0.1 & 0.7 & 44 & 1.6 & $\begin{array}{l}\text { microRNAs }^{g} \\
\text { endo-siRNA }\end{array}$ \\
\hline rde-1(ne300) & Argonaute & ex & 0.8 & 0.8 & 1.5 & 1.0 & \\
\hline rde-4(ne337) & dsRNA binding & ex & $\mathbf{0}$ & $\mathbf{0}$ & 2.7 & 1.7 & $\begin{array}{c}\text { endo-siRNA }^{\mathrm{h}} \\
\text { tncRNA }^{\mathrm{h}}\end{array}$ \\
\hline rde-3(ne298) & $\begin{array}{l}\text { Nucleotidyl- } \\
\text { transferase }\end{array}$ & ex, co, tr & 0.1 & $\mathbf{0}$ & 1.5 & 2.0 & $\begin{array}{c}\text { endo-siRNA }^{\mathrm{h}} \\
\text { tncRNA }^{\mathrm{h}}\end{array}$ \\
\hline rde-3(r459) & & & $\mathbf{0}$ & 0.3 & 0.5 & 0.7 & $\begin{array}{l}\text { mRNA repression }{ }^{i, j} \\
\text { endo-siRNA }^{\text {h }}\end{array}$ \\
\hline rde-2(ne221) & Novel & exgl, co, tr & 0.2 & $\mathbf{0}$ & 0.4 & 1.3 & $\begin{array}{c}\text { endo-siRNA } A^{\mathrm{h}} \\
\text { tncRNA }^{\mathrm{h}}\end{array}$ \\
\hline mut-7(pk204) & RNAse D & exgl, co, tr & 0.2 & 0.1 & 3.7 & 1.8 & $\begin{array}{c}\text { mRNA repression } \\
\text { endo-siRNA }^{\mathrm{h}} \\
\text { tncRNA }^{\mathrm{h}}\end{array}$ \\
\hline mut-14(pk738) & RNA helicase & exgl, tr & 0.1 & 0.3 & 0.5 & 1.6 & $\begin{array}{l}\text { mRNA repression } \\
\text { endo-siRNA }^{\mathrm{h}} \\
\text { mRNA repression }^{\mathrm{i}}\end{array}$ \\
\hline$r r f-1(p k 1417)$ & $\operatorname{RdRp}$ & exsom & 0.6 & 0.1 & 0.8 & 2.5 & $\begin{array}{c}\operatorname{tncRNA}^{\mathrm{h}} \\
\text { mRNA repression }\end{array}$ \\
\hline$r r f-3(p k 1426)$ & $\operatorname{RdRp}$ & eri & 0.1 & $\mathbf{0}$ & 2.3 & 1.9 & $\begin{array}{c}\text { endo-siRNA }{ }^{\mathrm{h}} \\
\operatorname{tncRNA}^{\mathrm{h}}\end{array}$ \\
\hline eri-1(mg366) & Exonuclease & eri & 0.2 & $\mathbf{0}$ & 4.9 & 2.4 & $\begin{array}{l}\text { mRNA repression }^{\mathrm{j}} \\
\text { endo-siRNA }^{\mathrm{h}} \\
\text { tncRNA }^{\mathrm{h}} \\
\text { mRNA repression }^{\mathrm{j}}\end{array}$ \\
\hline$r r f-2(o k 210)$ & $\operatorname{RdRp}$ & unknown & 0.6 & 0.7 & 4.7 & 2.6 & \\
\hline
\end{tabular}

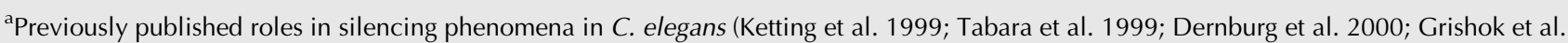
2000; Ketting and Plasterk 2000; Bernstein et al. 2001; Grishok et al. 2001; Ketting et al. 2001; Timmons et al. 2001; Simmer et al. 2002; Tabara et al. 2002; Tijsterman et al. 2002a; Kennedy et al. 2004; Chen et al. 2005; Tops et al. 2005). (ex) Exogenous RNAi of germline and somatic genes, (exgl) exogenous RNAi of germline genes preferentially, (exsom) exogenous RNAi of somatic genes preferentially, (eri) enhanced RNAi, (tr) transposon silencing, (co) cosuppression, (mir) microRNA biogenesis and function.

${ }^{b}$ Determined by Northern blot as described in Figure 1 and in Materials and Methods; Phosphorlmager signals were normalized to U6 levels in each sample and then expressed relative to the wild type. Values in boldface type are those at least fivefold elevated or decreased compared with the wild type. This quantitative cutoff was chosen to capture a subjective judgment of significantly abnormal signals.

"Measurement shown is for Ct1189; similar results were obtained for Ct1182 (except that only Ct1189 was assayed in eri-1 total RNA).

dMeasurement shown is for tncR35; similar results were obtained for tncR36 and for tncR4 (except that tncR36 and tncR4 were not assayed in eri-1).

${ }^{\mathrm{e}}$ Measurement shown is for mir-238; similar results were obtained for mir-38, mir-42, mir-52, mir-58, mir-71, mir-79, and mir-229 (except that only mir-238 was assayed in eri-1 total RNA).

${ }^{\mathrm{f}}$ Based on interpretation of results presented in this paper.

${ }^{g}$ Based on accumulation of pre-miRNA (Figure 1).

${ }^{\mathrm{h}}$ Small RNAs reduced significantly, as indicated by the data in Figure 1 (and summarized in this table).

'Endogenous messenger RNA elevated, as indicated by the data in Figure 2.

'Endogenous messenger RNAs elevated, as indicated by the data in Figure 3 and Supplemental Table S1.

et al. 2005; Lu et al. 2005; Wilkins et al. 2005), the full range of roles for endogenous RNAi in normal cells is still emerging. In addition to miRNAs, cDNA cloning has identified other noncoding small RNAs in C. elegans, including the tiny noncoding RNAs (tncRNAs), whose functions are yet unknown (Ambros et al. 2003). C. elegans also contains a surprisingly abundant population of apparent siRNAs from a wide variety of protein-coding genes, suggesting that endogenous RNAi- mediated gene regulation occurs on a genomic scale in normal worms (Ambros et al. 2003).

Here we report evidence in support of multiple pathways for widespread, endogenous RNAi in C. elegans. We show that both protein-coding and noncoding sequences are involved in ongoing RNAi in C. elegans and that the mRNA levels of many diverse protein-coding genes of C. elegans are affected, in some cases profoundly, by endog- 
enous gene silencing. We show that endogenous RNAi pathways share core components with exogenous RNAi but that these pathways also differ in their requirements for certain more specialized factors. In particular, we find that $r r f-3$ mutants and eri-1 mutants, which are enhanced for exogenous RNAi (Simmer et al. 2002; Kennedy et al. 2004) and enhanced for transgene-driven endogenous RNAi (Grishok et al. 2005; Robert et al. 2005), are nevertheless defective in silencing of many endogenous transcripts and in the production of certain endogenous siRNAs and tncRNAs. We suggest that the enhanced RNAi phenotype (eri) of rrf-3 and eri1 mutants reflects a competition between specialized silencing pathways for core components of the RNAi machinery.

\section{RESULTS}

\section{Similar genetic requirements for endogenous siRNAs and tncRNAs}

We previously described the identification of three major classes of endogenously encoded small RNAs among cDNAs cloned from size-fractionated, C. elegans total RNA (Ambros et al. 2003). In addition to microRNAs, these cDNAs included a second class of $\sim 22$-nt RNAs that are apparently derived from noncoding sequences, which we termed tncRNAs. tncRNAs are distinct from microRNAs because tncRNAs do not appear to be processed from short hairpin precursors. A third, and abundant, class of small cDNAs from C. elegans consists of apparent siRNAs that are precise anti-sense matches to confirmed or predicted protein-coding genes (Ambros et al. 2003). For this study, we analyzed the sequences of additional cDNA clones from wild-type C. elegans, and these additional sequences, combined with the previous data, comprise approximately three dozen different tncRNAs and 1799 distinct siRNAs derived from 1085 genes (See supplemental Table S1 at http:// chronic.dartmouth.edu/VRA/Lee_etal_2006_Table_S1).

To confirm that the cloned siRNA cDNAs represent bona fide endogenous silencing by RNAi, and to characterize the relationship between tncRNAs and siRNAs, we tested whether the accumulation of endogenous siRNAs and tncRNAs depends on known components of the RNAi machinery. Accordingly, total RNA was prepared from mixed-stage populations of wild-type nematodes and from RNAi-defective mutants $r d e-1, r d e-2, r d e-3$, mut-7, mut-14, $r r f-1$, and $d c r-1$ (see Table 1 for references). We also prepared RNA from mutants enhanced for RNAi, rrf-3, and eri-1, and animals depleted for $a l g-1$ and $a l g-2$, which encode Argonaute proteins required for the accumulation of microRNAs (Grishok et al. 2001). These RNA samples were analyzed by Northern blotting with probes complementary to cloned tncRNAs and siRNAs. Most siRNA probes did not produce a detectable signal in mutants or in wild-type RNA, indicating low absolute abundance of any one particular siRNA sequence. However, for the siRNAs $\underline{\mathrm{Ct}}$ (Cloned transcript)1182 and

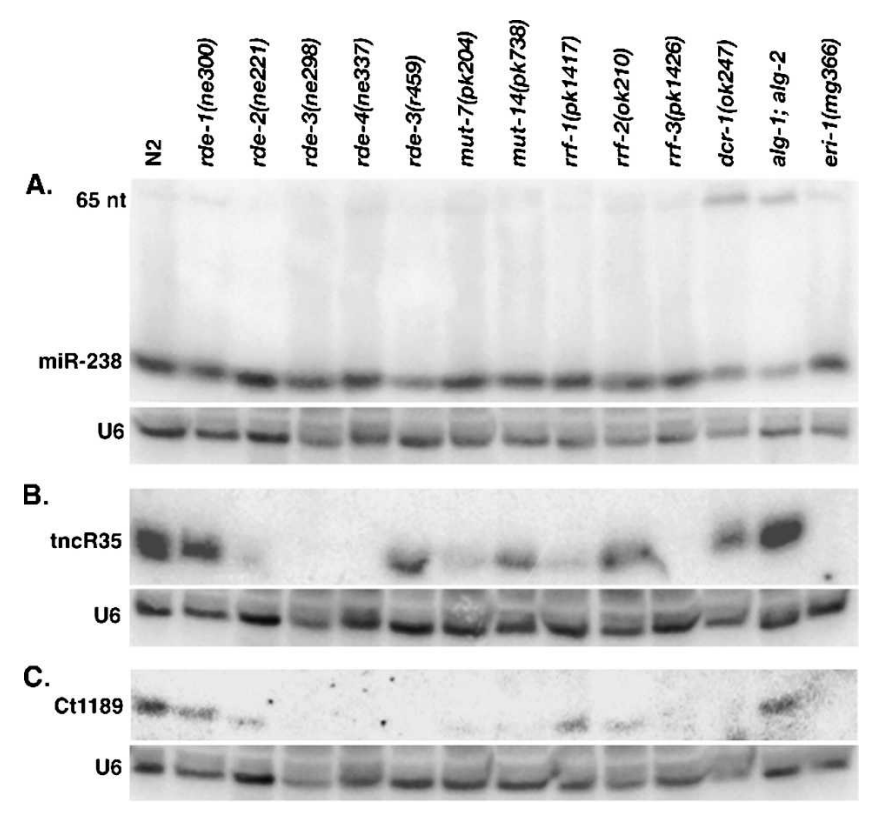

FIGURE 1. Northern blot hybridization analysis of $(A)$ a representative microRNA (miR-238), (B) tncRNA (tncR35), and (C) siRNA (Ct1189, from protein-coding gene C44B11.6) in total RNA from the wild type and mutants defective in components of RNAi pathways. Each lane of a $12.5 \%$ acrylamide urea gel was loaded with $25 \mu \mathrm{g}$ of total RNA. Loading was calibrated by hybridizing U6 snRNA probe to each filter after completion of the small RNA analysis. See Table 1 for quantitative analysis.

Ct1189 (complimentary to predicted gene C44B11.6 transcript), hybridizing signals of $\sim 22 \mathrm{nt}$ in length were detected (Fig. 1C; data not shown). The Ct1182 and Ct1189 siRNAs were significantly reduced or absent in mutants of $r d e-2, r d e-3$, rde-4, mut-7, mut-14, and dcr-1 (Fig. 1C; Table 1), which clearly distinguishes Ct1182 and Ct1189 from microRNAs, which are unaffected by all the mutants except $d c r-1$ and $a l g$ 1(RNAi); alg-2(0) (Fig. 1A; Table 1). These results are consistent with findings for siRNAs produced from exogenous dsRNA (Tabara et al. 2002; Tijsterman et al. 2002a; Tops et al. 2005), supporting the conclusion that Ct1182 and Ct1189 are bona fide siRNAs produced from an endogenous gene (in this case C44B11.6). Exogenous siRNAs have been shown to accumulate in mut-14 mutants (Tijsterman et al. 2002a), so the decreased signal for Ct1189 in mut-14(pk738) (Fig. 1C) may indicate differences in the biogenesis of at least some endogenous siRNAs compared with exogenous siRNAs.

Also consistent with divergent pathways for endogenous siRNAs and exogenous siRNAs, we found that $r r f-3$ and eri-1 mutants showed decreased level of the Ct1189 and Ct1182 hybridization (Fig. 1C; Table 1; data not shown). The absence of these endogenous siRNAs in $r r f-3$ and eri-1 mutants is in striking contrast to the fates of exogenously derived siRNAs. Exogenously derived siRNAs are more abundant in eri-1 and $r r f-3$ mutants compared with the wild type, consistent with the enhanced exogenous RNAi phenotypes of rrf-3 and eri-1 (Simmer et al. 2002; Kennedy et al. 2004). 
tncRNA probes produced similar results to those of endogenous siRNAs. tncRNA signals were reduced in many of the same mutants where Ct1182 and Ct1189 were also reduced (Fig. 1B,C; Table 1; data not shown). This indicates that the tncRNAs, although originating from noncoding loci in the $C$. elegans genome, are produced by mechanisms similar to those that generate endogenous and exogenous siRNAs from protein-coding sequences, and distinct from microRNAs. There are some apparent differences between the biogenesis of tncRNAs and the endogenous siRNAs that we assayed. For example, in mut-14(pk738) tncR35 was easily detected, which is generally consistent with previous findings for exogenous siRNAs (Tijsterman et al. 2002a), while the siRNA Ct1189 signal was much more reduced in mut-14(pk738) than was tncR35. Also, tncRNAs were also easily detectable (at reduced levels) in RNA from rde-3(r459) (formerly mut-2) but undetectable in rde-3(ne298), unlike the siRNAs, Ct1182 and Ct1189, which were undetected in both these $r d e-3$ mutants (Fig. 1C; Table 1; data not shown).

\section{mRNAs are elevated in silencing mutants}

If the siRNAs produced in normal worms represent ongoing gene silencing, then at least some of the genes being silenced should exhibit elevated levels of mRNA in silencing mutants. We tested this hypothesis by Northern blot and by microarray hybridization using total RNA from silencing mutants. Probe from one of the genes represented by multiple, independent siRNAs in our cDNA libraries, C04F12.9 (six distinct cDNA sequences cloned; see Table S1 at http://chronic.dartmouth.edu/VRA/Lee_etal_2006_ Table_S1), hybridized to a transcript of the predicted fulllength C04F12.9 mRNA in rde-2, rde-3, mut-7, and mut-14 mutants but registered no detectable signal in RNA from other silencing mutants, including alg-1(RNAi); alg-2(0), or from wild-type animals (Fig. 2). This supports the conclusion that $r d e-2, r d e-3, m u t-7$, and mut-14, which are known to encode factors required for the silencing of transposons in C. elegans (Ketting et al. 1999; Tabara et al. 1999; Tijster-
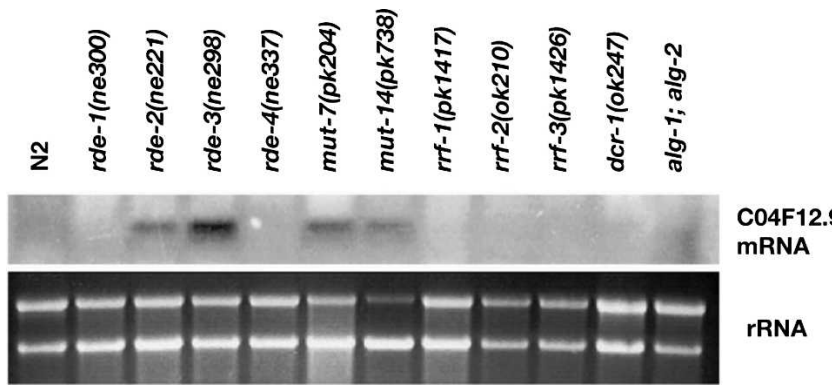

FIGURE 2. Northern blot hybridization analysis of C04F12.9 mRNA expression in total RNA from the wild type and mutants defective in components of RNAi pathways. Each lane of a 1\% agarose formaldehyde gel was loaded with $20 \mu \mathrm{g}$ of total RNA. Approximately equal loading was confirmed by ethidium bromide staining of the gels before transfer (bottom). man et al. 2002a; Vastenhouw et al. 2003), also regulate C04F12.9 transcript levels. This suggests that the same pathway that silences transposons also silences nontransposon genes such as C04F12.9. Interestingly, C04F12.9 transcript levels are not elevated in rde-4(ne337) RNA, although the siRNAs from a different gene, C44B11.6 (siRNAs Ct1182 and Ct1189) (Fig. 1; Table 1), were strongly reduced in rde4(ne337). This suggests that not all endogenous transcripts are silenced by precisely the same mechanisms.

Microarray hybridization experiments (Fig. 3; Table 2; see Table S1 at http://chronic.dartmouth.edu/VRA/Lee_etal_2006_Table_S1) further support the hypothesis that diverse genes are silenced by RNAi mechanism in wild-type worms. For the 17,774 transcripts analyzed, specific subsets of transcripts were elevated at least twofold in RNA from each mutant compared with RNA from the wild type. Specifically, $221,293,129,274$, and 257 transcripts were elevated at least twofold in $r r f-1, r r f-2, r r f-3$, eri-1, and $r d e-3(r 459)$ mutants, respectively (Fig. 3A; see Table $\mathrm{S} 1$ at http://chronic. dartmouth.edu/VRA/Lee_etal_2006_Table_S1). Similar, but generally lesser, numbers of mRNAs $(104,86,136,179$, and 93, respectively) were reduced twofold or more in these mutants. The vast majority of transcripts $(>90 \%$ for all mutants) changed less than twofold in either direction in all the mutants. This indicates that in these mutants, most mRNAs remain essentially unchanged in absolute levels, with the exception of several hundred mRNAs $(<10 \%$ of the total) that were either elevated or reduced at least twofold. A notable subset of these genes are 225 genes that were elevated fivefold or more in at least one mutant (Fig. 3A; see Table S1 at http://chronic.dartmouth.edu/VRA/Lee_etal_ 2006_Table_S1).

The finding that certain mRNA transcripts are elevated substantially in $r d e-3$ and $r r f-1$ mutants, which are known to be deficient in RNAi components, supports the idea that these elevated transcripts ordinarily are subjected to potent inhibition by endogenous RNAi. However, these data alone do not distinguish whether these elevated genes are directly affected by RNAi that is triggered by complementary double-stranded RNA or are elevated as an indirect consequence of the physiology of the $r d e-3$ or $r r f-1$ mutants. In support of the direct involvement of gene silencing in the regulation of at least some of the elevated mRNAs, we identified, by cDNA cloning, siRNAs present in wild-type worms that were precisely complementary to transcripts elevated in RNAi-defective mutants (see Table S1 at http: //chronic.dartmouth.edu/VRA/Lee_etal_2006_Table_S1). Moreover, genes from which complementary siRNAs were cloned were significantly enriched among the transcripts elevated at least twofold in rde3(ne298), rrf-1(pk1417), or eri-1(mg366) mutants, and among transcripts elevated at least 3.1-fold in $r r f-3$ (pk1426) mutants (Table 2). We interpret these results to indicate that $r d e-3$, eri-1, rrf-3, and $r r f-1$ normally function in RNAi pathways that substantially attenuate the expression of a number of endogenous genes. We did not find an enrichment for siRNA- 

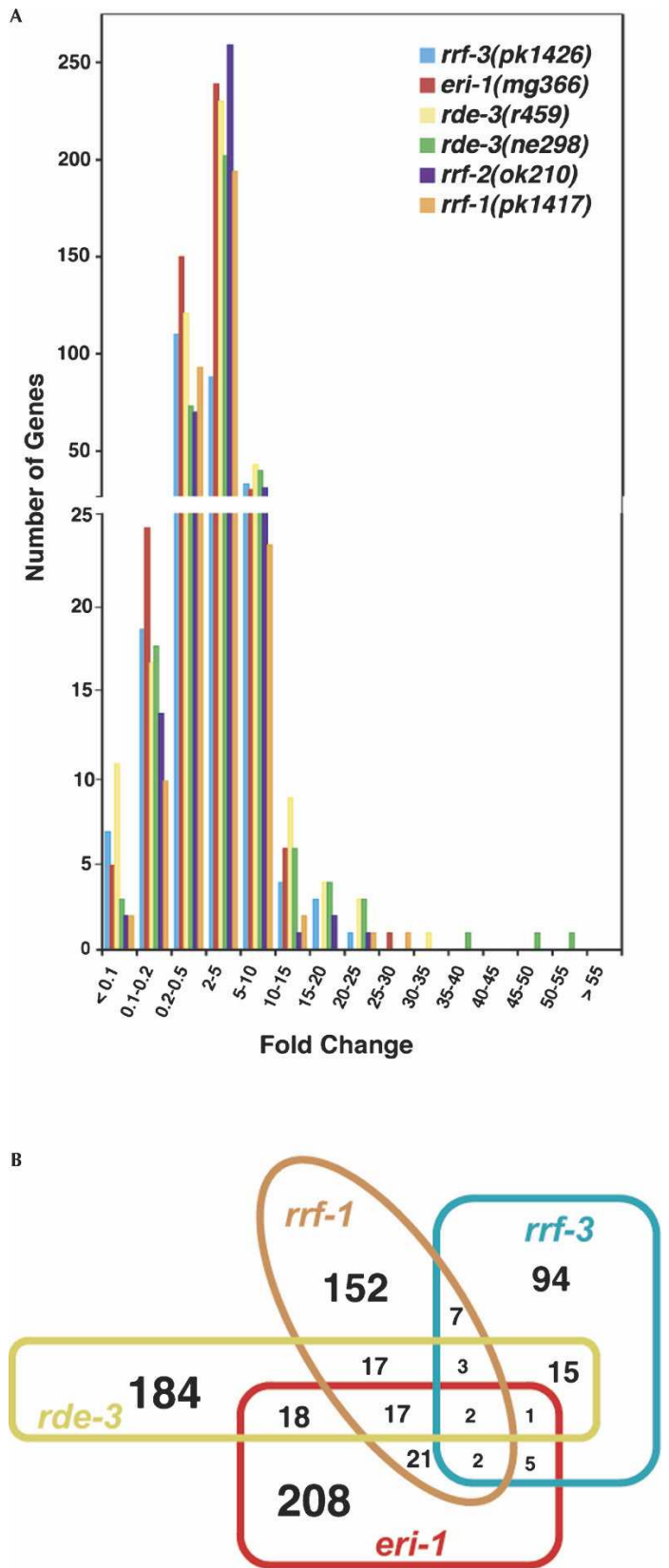

FIGURE 3. (A) Distribution of changes in mRNA levels in six mutant populations compared with the wild type for 17,774 C. elegans genes, as assayed by microarray hybridization (see Materials and Methods). Only transcripts that increased or decreased at least twofold are shown. (B) Venn diagram of genes elevated twofold or more in mutants of rrf3(pk1426) (129 genes), rrf-1(pk1417) (221 genes), rde-3(ne298) (257 genes), and eri-1(mg366) (274 genes).

generating genes among the top genes elevated in $r r f-2$ mutants or in $r d e-3(r 459)$ (see Table S1 at http://chronic.dartmouth.edu/VRA/Lee_etal_2006_Table_S1). These findings suggest that the $r d e-3$ (ne298) mutation may affect $r d e-3$ activity more strongly than does $r d e-3(r 459)$. These results also suggest that RRF-2 may not play a major role in endogenous RNAi, although it is also possible that RRF-2 could be involved in the silencing of a relatively limited set of endogenous genes, and our data may be too incomplete to fully test for a correlation between elevated mRNA level in $r r f-2$ mutants and cloned siRNAs.

\section{Partially overlapping sets of genes are affected in different silencing mutants}

The sets of endogenous mRNAs that were affected in RNAidefective mutants differed substantially among the individual mutants that we tested. For example, of 274 transcripts elevated at least twofold in eri-1 mutants, and the 129 transcripts elevated at least twofold in $r r f-3$ mutants, only 10 were elevated in both mutants (Fig. 3B). Only two genes (Y17G 7B.19 and F39E9.7) were elevated at least twofold in all four mutants of eri-1, rde-3, rrf-2, and rrf-3 (see Table S1 at http:// chronic.dartmouth.edu/VRA/Lee_etal_2006_Table_S1).

\section{DISCUSSION}

\section{Endogenous silencing of protein-coding and noncoding sequences in normal worms}

The results reported here support the conclusion that the small endogenous RNAs that are anti-sense to transcripts encoding diverse proteins in wild-type worms (Ambros et al. 2003) are indeed the consequence of ongoing RNAi of endogenous mRNAs. Two observations support this conclusion: First, the accumulation of certain siRNAs is reduced in mutants defective in RNAi machinery. Second, in the silencing mutants eri-1, rrf-1, rrf-3, and $r d e-3$ (although not for $r r f-2$ mutants), the set of substantially elevated transcripts was enriched for genes from which siRNAs were cloned. This correlation between the occurrence of endogenous siRNAs and the de-repression of the corresponding mRNAs in eri-1, $r r f-1, r r f-3$, and $r d e-3$ mutants suggests that the ERI-1, RRF1, RRF-3, and RDE-3 gene products directly inhibit the accumulation of particular mRNAs in wild-type worms.

Not all of the genes from which siRNAs were cloned exhibited elevated gene expression in the silencing mutants that we analyzed, and siRNAs were not cloned from some genes that were substantially elevated in one or more mutants. Our siRNA cDNA library cloning efforts are likely not saturated, which could explain the absence of detectable siRNAs from some bona fide silenced genes. There are also numerous possible explanations for the apparent lack of change in the levels of some of the genes from which siRNAs were cloned. For example, genes that are attenuated by endogenous RNAi in only a subset of cells that express the gene, or only at a limited stage of development, might not exhibit a detectable change over background in mixedstage populations of mutants.

We find that tncRNAs are produced from noncoding sequences by RNAi-related mechanisms similar to those 
TABLE 2. Enrichment of genes with cloned siRNAs among genes elevated in mutants.

\begin{tabular}{lcccc}
\hline & \multicolumn{4}{c}{ Mutants } \\
\cline { 2 - 5 } & rde-3(ne298) & rrf-3(pk1426) & rrf-1(pk1417) & eri-1(mg366) \\
\hline $\begin{array}{c}\mathrm{T}=\text { Threshold (fold } \\
\text { wild-type level) }\end{array}$ & 2.0 -fold & 3.1 -fold & 2.0 -fold & 2.0 -fold \\
$\begin{array}{c}\mathrm{M}=\text { mRNAs above } \\
\text { threshold }\end{array}$ & 257 & 76 & 221 & 274 \\
$\begin{array}{c}\mathrm{S}=\text { siRNAs cloned from } \\
\text { genes above threshold T }\end{array}$ & 99 & 18 & 91 & 59 \\
$\begin{array}{c}\text { siRNAs/gene (S/M) } \\
P \text { value }\end{array}$ & 0.39 & 0.24 & 0.41 & 0.21 \\
\hline
\end{tabular}

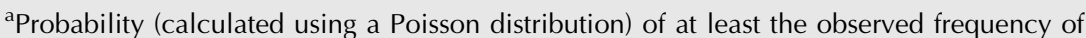
siRNAs per gene for the genes above threshold $\mathrm{T}$, given the average siRNAs per gene for all genes on the array (1799 siRNAs were cloned from a total of 17,774 genes $=0.10$ siRNAs/ gene). For all these mutants except for rrf-3(pk1426), the set of mRNAs increased at least twofold were significantly enriched for siRNAs; for $r$ rf-3(pk1426), a $P$ value cutoff of 0.05 was satisfied for the set of mRNAs increased at least 3.1-fold.

eri-1, and rde-3 (Ketting et al. 1999; Simmer et al. 2002; Tijsterman et al. 2002a; Kennedy et al. 2004; Chen et al. 2005).

Our data do not address whether endogenous RNAi in C. elegans is exerted post-transcriptionally, for example, by siRNA-guided mRNA turnover, or transcriptionally, for example, by siRNAguided chromatin modification. The siRNAs analyzed in this study preferentially originate from exonic sequences (Ambros et al. 2003; data not shown), so these could reflect mRNA-level post-transcriptional RNAi. However, some small RNAs were cloned that correspond to introns and therefore may be involved in targeting the destruction of pre-mRNA (Bosher et al. 1999). Further, the tncRNAs

that produce endo-siRNAs. A hallmark of tncRNAs that apparently distinguishes them from most endo-siRNAs is that many are produced in sufficient abundance to be easily detected by Northern blot hybridization (Ambros et al. 2003). Furthermore, they are anti-sense to regions of the C. elegans genome that have not been previously identified as transcriptionally active. These tncRNA sequences may accumulate because they happen to be intrinsically stable, because they represent particularly good substrates for RdRp, and/or because they may be specifically incorporated into RNP complexes of as-yet-unknown function. We do not know the function of tncRNAs, but the observation that they share essentially the same genetic requirements for their production as siRNAs suggests that they may also function as guide RNAs for gene silencing, perhaps by targeting the chromatin from which they originate or by targeting mRNAs from other loci, analogous to microRNAs or trans-acting siRNAs (Peragine et al. 2004; Vazquez et al. 2004; Allen et al. 2005).

\section{The levels of many messenger RNAs are attenuated by endogenous RNAi in wild type worms}

Although transposon sequences were among the transcripts elevated in silencing mutants and from which siRNAs were cloned, the majority of the affected genes were not parts of transposons (Ambros et al. 2003). Therefore, these results support an expanded role for endogenous RNAi mechanisms in normal worms to include genes involved in worm development and/or physiology. Some genes were elevated 30 - to 40-fold compared with wild type, which indicates that endogenous silencing pathways could contribute significantly to the level of gene expression across the genome. Misexpression of diverse genes could account for the visible phenotypes, including sterility and lethality, exhibited by most silencing mutants, including mut-7, mut-14, rrf-3, come from sequences upstream or downstream of coding sequence and hence could function in transcriptional silencing by RNAi-guided chromatin modification. Consistent with the latter possibility, evidence has been reported for RNAi-mediated silencing of a somatic gene on the level of transcription (Grishok et al. 2005), and components of chromatin modifying machinery have been found to function in RNAi pathways in C. elegans (Kim et al. 2005; Robert et al. 2005).

\section{Multiple pathways for endogenous silencing}

It is striking that the sets of genes substantially affected in $r r f-3$, eri-1, $r r f-1$, and $r d e-3$ mutants were only partially overlapping. This observation suggests complex endogenous gene-silencing pathways in C. elegans, analogous to the multiple, functionally diverse small RNA pathways observed in plants (Xie et al. 2004) and the apparently distinct mechanisms for RNAi-mediated transposon silencing in Dictyostelium discoideum (Kuhlmann et al. 2005). Individual C. elegans genes appear to be differentially sensitive to different endogenous RNAi mechanisms. For some genes, this specificity could reflect tissue specific silencing; for example, $r r f-1$ is thought to be primarily involved in silencing somatic genes, and rrf-3 may act more broadly (Simmer et al. 2002). Thus, germline genes might be expected to be sensitive to mutation of $r r f-3$ but not of $r r f-1$. Furthermore, some genes may be subjected to endogenous silencing on the transcriptional level, while others may be post-transcriptionally repressed, and these processes could involve distinct, specialized components or cellular compartments.

Endogenous silencing pathways may also diverge to accommodate various modes of generating, maintaining, and/or trafficking (Winston et al. 2002) different classes of double-stranded RNAs and/or $\sim 22-n t$ RNAs. In this regard, there is evidence for distinct classes of tncRNAs and endosiRNAs based on differing requirements for $r d e-4$ activity. 
Duchaine et al. (2006) report that $r d e-4$ was required for accumulation of tncRNAs from the "X-cluster" locus but not for other tncRNAs and endo-siRNAs that they examined. We found that $r d e-4$ is required not only for X-cluster tncRNAs (tncR34 and tncR36) but also for tncR4, which is not located in the X-cluster (Ambros et al. 2003), and also for the particular siRNAs that we assayed (Fig. 1; Table 1). These results suggest the presence of RDE-4-dependent and RDE-4-independent mechanisms for the production of distinct classes of endogenous siRNAs and tncRNAs.

\section{Compromising some endogenous RNAi pathways can enhance other RNAi pathways}

Animals mutant for the RdRp gene $r r f-3$, or the nucleaseencoding gene eri-1, exhibit an eri phenotype, in that they are more sensitive than wild type to gene inactivation by feeding or injection of double-stranded RNA (Simmer et al. 2002; Kennedy et al. 2004). The enhancement is not restricted only to exogenous RNAi, as $r r f-3$ and eri- 1 mutants are also enhanced for the silencing of repetitive transgenes through RNAi pathways elicited without an external source of double-stranded RNA (Grishok et al. 2005; Robert et al. 2005). We observed that the accumulation of a specific siRNA or tncRNA can depend on the wild-type activity of $r r f-3$ or eri-1, and that numerous endogenous genes are up-regulated in these mutants. The finding that $r r f-3$ and eri-1 are involved in ongoing silencing of endogenous sequences supports the hypothesis that the enhancement of some RNAi pathways in these mutants could be a consequence of reduced activity of other endogenous RNAi pathways, resulting in elevation of critical components of the RNAi machinery.

An elevation in RNAi components after loss of endogenous silencing could occur by numerous possible mechanisms. In one model, loss of endogenous silencing of mRNAs that encode limiting protein components of the core RNAi machinery could lead to increased levels of those proteins, analogous to the regulation of Dicer-Like-1 in Arabidopsis by a microRNA (Xie et al. 2003). This first model would predict that one or more mRNAs encoding proteins required for exogenous RNAi would be elevated in $r r f-3$ or eri-1 mutants. Our data do not offer strong support for this model: Of 209 genes reported to function in C. elegans RNAi pathways (Vastenhouw et al. 2003; Grishok et al. 2005; Kim et al. 2005; Robert et al. 2005), none were observed to increase in mRNA level more than twofold in rrf-3, and only two, F37B12.4 and F14H3.12, were observed to increase in mRNA level more than twofold in eri-1 (2.65-fold and 2.28fold, respectively) (see Table S1 at http://chronic.dartmouth. edu/VRA/Lee_etal_2006_Table_S1). In an alternative model, similar to one advanced by Duchaine et al. (2006), loss of ERI-1- or RRF-3-mediated silencing could liberate core RNAi proteins that are otherwise ordinarily dedicated to endogenous silencing in complexes with RRF-3 and/or ERI-1 (Fig. 4). These hypothetical limiting RNAi factors, whose redeploy-
A. wild type
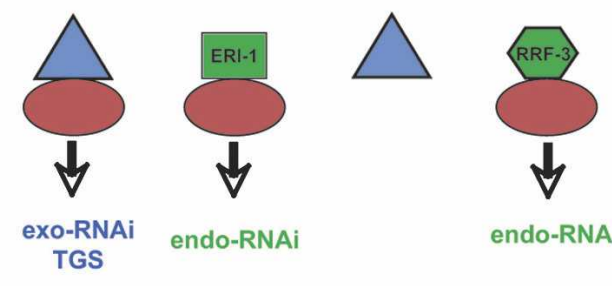

endo-RNAi

B. eri-1 mutant
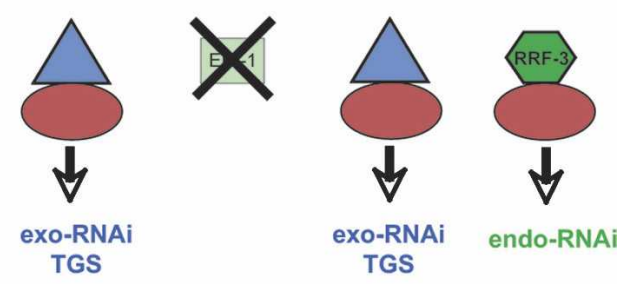

C. rrf-3 mutant
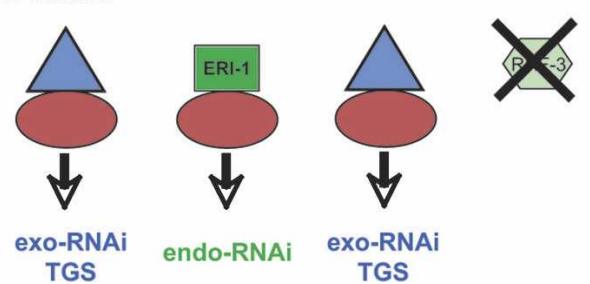

FIGURE 4. A model for the enhancement of certain RNAi processes in $r r f-3$ and eri- 1 mutants, based on our observations that $r r f-3$ and eri-1 animals exhibit defects in certain endogenous RNAi processes, including accumulation of tncRNAs (Fig. 1; Table 1) and repression of endogenous genes (Fig. 3; Table 2; see Table S1 at http://chronic. dartmouth.edu/VRA/Lee_etal_2006_Table_S1). (A) In the wild type, multiple RNAi pathways are engaged in silencing elicited by nuclear transcription (e.g., endo-RNAi and transcriptional gene silencing [TGS]) or by exogenously supplied dsRNA. All of these pathways involve certain core RNAi components (represented by the red oval) (Tabara et al. 1999; Grishok et al. 2005; Kim et al. 2005), while more specialized components (represented by triangles, square, and hexagon) are involved in distinct pathways for silencing of subsets of endogenous genes, TGS, and/or exogenous RNAi. In this model, specialized factors for exo-RNAi/TGS (triangles) are posited to be in excess. (B) In eri-1 mutants, the loss of ERI-1 protein activity disables a branch of endogenous RNAi, leading to the liberation of core components (red oval) that then supplement complementary pathways (in this case, exogenous RNAi and TGS). (C) In rrf-3 mutants, the loss of RRF-3 protein activity similarly disables another branch of endogenous RNAi, leading to the liberation of core components that then supplement complementary pathways.

ment would underlie the enhanced RNAi of eri-1 and $r r f-3$ mutants, could include the core RNAi proteins shown to interact with RRF-3 and/or ERI-1 in vivo (Duchaine et al. 2006). In further support of this model, reduction of certain endogenous RNAi factors other than ERI-1 and RRF-3 can also result in an eri loss-of-function phenotype (Duchaine et al. 2006).

These results imply substantial cross-regulatory interactions among silencing processes in C. elegans. If core RNAi components are ordinarily limiting in wild-type worms, 
then a load on one RNAi pathway can impact the efficacy of other RNAi pathways. For example, an exogenous RNAi challenge may deplete components required for endogenous RNAi, and lead to up-regulation of silenced endogenous genes. Such cross-regulation could permit the cell and/ or organism to monitor, and perhaps coordinate, the regulation of diverse RNAi processes.

\section{MATERIALS AND METHODS}

\section{Nematode strains and culture}

Methods for nematode culture and handling were as described (Wood 1988). Strains used were N2 Wild-type C. elegans, Bristol variant; VT1017 rde-1(ne300); WM29 rde-2(ne221); WM30 rde3(ne298); VT1020 rde-4(ne337); NL739 rde-3(r459) (formerly mut2) (Chen et al. 2005); NL917 mut-7(pk204); NL1838 mut-14(pk738); NL2098 rrf-1(pk1417); EL476 rrf-2(ok210); NL2099 rrf-3(pk1426); WM53 alg-2(ok304); GR1373 eri-1(mg366). Dicer-depleted animals were obtained by ivermectin selection of homozygous dcr-1(ok247) progeny from worms of genotype avr-14(ad1302); avr-15(ad1051) glc-1(pk54::Tc1); dcr-1(ok247)/qC1 [neIs(myo2::avr-15,rol-6,unc22 (RNAi))] (C. Mello, pers. comm.). alg-1 depleted RNA samples were obtained by subjecting a mixed population of alg-2(ok304) animals to a bacterial food source expressing a dsRNA corresponding to an 380 -nt NruI fragment of alg-1 genomic sequence (pCMH530) (Timmons et al. 2001). Populations of wild-type and mutant worms were examined by microscopy to confirm the presence of approximately similar fractions of embryos, larvae, and adults in all samples. A similar representation of developmental stages was achieved in samples of all of the mutants, except for dcr-1- and alg-depleted animals, which were generally enriched for adults relative to other stages.

\section{Preparation of worm RNA}

Mixed populations of C. elegans larvae, embryos, and adults were grown on agar plates containing lawns of Escherichia coli OP50, harvested, and frozen at $-80^{\circ} \mathrm{C}$, and total RNA was extracted using the Trizol method as described previously (Lee and Ambros 2001).

\section{Northern blot assays}

For detection of small RNAs (Fig. 1; Table 1), total RNA was fractionated on denaturing $12.5 \%$ acrylamide urea gels, transferred electrophoretically to Genescreen Plus hybridization membranes, and probed with ${ }^{32} \mathrm{P}$ oligonucleotide probes labeled using the Starfire system (Integrated DNA Technologies) as described previously (Lee and Ambros 2001). Hybridized signals were detected using a Molecular Dynamics Storm PhosphorImager and quantified using ImageQuant software. Oligonucleotide probes were of the following sequences: mir-238, CTGAATGGCATCGGAGTACAAA; miR-38, CCAGTTTTTCTCCCGGTGATAGAGA; miR-42, GTA GATGTTA ACCCGGTGA; miR-52, AGCACGGAAACATATGTACGGGTG; miR-58, ATTGCCGTACTGAACGATCTCA; miR-71, CACTACCC ATGTCTTTCA; miR-79, AGCTTTGGTAACCTAGCTTTAT; miR229, GATGGAAAAGATAACCAGTGTCATT; tncR35, GAAAAGTG AAACTTCTTTAC; tncR4, TGCAACGG AACACGACTAAC; tncR7,
CAGCCTACGGAATGGTTGTC; siRNA Ct1189, TTCACAAGATA TGAACTTC; siRNA Ct1182, ACAAGACTGCAATATCCTC; U6, TGTCATCCTTGCGCA.

For detection of mRNAs (Fig. 2), $20 \mu \mathrm{g}$ of total worm RNA was fractionated on denaturing $1 \%$ agarose formaldehyde gels, transferred by capillary transfer to nitrocellulose hybridization membranes, and probed with ${ }^{32} \mathrm{P}$ riboprobe probe complementary to the C04F12.9 transcript, derived from T7 transcription (Ambion MaxiScript) of SacI-digested pCMH564.

\section{Microarray analysis}

Wild-type or mutant worms were cultured in triplicate, and independent samples of total RNA were prepared using the same method as for Northern blotting (see above). RNA was labeled and hybridized to Affymetrix C. elegans microarrays according to the manufacturer's instructions. Hybridization signals above background for each gene were normalized across all arrays. A t-test (5\% confidence) was applied to the triplicate sample data for each transcript in each mutant to identify genes significantly elevated or decreased compared with the wild type. The average of the triplicate samples was then calculated for each gene and worm strain, and the ratio of the level of expression in the mutant to the level of expression in the wild type was calculated as the ratio of the averages. Supplemental Table S1 (http://chronic.dartmouth.edu/ VRA/Lee_etal_2006_Table_S1) is a spreadsheet containing the microarray data in unprocessed and processed form, along with gene annotations and other materials used in the analysis.

\section{ACKNOWLEDGMENTS}

We thank Ausra Milano of the Dartmouth Microarray Core Facility for microRNA hybridization and data collection, members of the Ambros laboratory for stimulating discussions, and T. Duchaine and C. Mello for sharing data prior to publication. C.M.H. was supported by a NIH Postdoctoral Fellowship F32GM69186-1. This work was supported by Public Heath Service NIH grant (GM34028) to V.A. Some of the strains used in this study were obtained from the Caenorhabditis Genetics Center, which is supported by the NIH National Center for Research Resources.

Received September 14, 2005; accepted December 14, 2005.

\section{REFERENCES}

Allen, E., Xie, Z., Gustafson, A.M., and Carrington, J.C. 2005. microRNA-directed phasing during trans-acting siRNA biogenesis in plants. Cell 1: 207-221.

Ambros, V. 2004. The functions of animal microRNAs. Nature 431: 350-355.

Ambros, V., Lee, R.C., Lavanway, A., Williams, P.T., and Jewell, D. 2003. MicroRNAs and other tiny endogenous RNAs in C. elegans. Curr. Biol. 13: 807-818.

Baulcombe, D. 2004. RNA silencing in plants. Nature 431: 356-363.

Bernstein, E., Caudy, A.A., Hammond, S.M., and Hannon, G.J. 2001. Role for a bidentate ribonuclease in the initiation step of RNA interference. Nature 409: 363-366.

Bosher, J.M., Dufourcq, P., Sookhareea, S., and Labouesse, M. 1999. RNA interference can target pre-mRNA: Consequences for gene expression in a Caenorhabditis elegans operon. Genetics 153: 1245-1256. 
Chen, C.C., Simard, M.J., Tabara, H., Brownell, D.R., McCollough, J.A., and Mello, C.C. 2005. A member of the polymerase $\beta$ nucleotidyltransferase superfamily is required for RNA interference in $C$. elegans. Curr. Biol. 15: 378-383.

Dernburg, A.F., Zalevsky, J., Colaiacovo, M.P., and Villeneuve, A.M. 2000. Transgene-mediated cosuppression in the C. elegans germ line. Genes \& Dev. 14: 1578-1583.

Duchaine, T.F., Wohlschlegel, J.A., Kennedy, S., Bei, Y., Conte, D., Pang, K., Brownell, D.R., Harding, S., Mitani, S., Ruvkun, G., et al. 2006. Functional proteomics reveals the biochemical niche of $C$. elegans DCR-1 in multiple small-RNA-mediated pathways. Cell 124: $343-354$

Fagard, M., Boutet, S., Morel, J.B., Bellini, C., and Vaucheret, H. 2000. AGO1, QDE-2, and RDE-1 are related proteins required for posttranscriptional gene silencing in plants, quelling in fungi, and RNA interference in animals. Proc. Natl. Acad. Sci. 97: 11650-11654.

Grishok, A., Tabara, H., and Mello, C.C. 2000. Genetic requirements for inheritance of RNAi in C. elegans. Science 287: 2494-2497.

Grishok, A., Pasquinelli, A.E., Conte, D., Li, N., Parrish, S., Ha, I., Baillie, D.L., Fire, A., Ruvkun, G., and Mello, C.C. 2001. Genes and mechanisms related to RNA interference regulate expression of the small temporal RNAs that control C. elegans developmental timing. Cell 106: 23-34.

Grishok, A., Sinskey, J.L., and Sharp, P.A. 2005. Transcriptional silencing of a transgene by RNAi in the soma of C. elegans. Genes \& Dev. 19: 683-696.

Kennedy, S., Wang, D., and Ruvkun, G. 2004. A conserved siRNAdegrading RNase negatively regulates RNA interference in C. elegans. Nature 427: 645-649.

Ketting, R.F. and Plasterk, R.H. 2000. A genetic link between co-suppression and RNA interference in C. elegans. Nature 404: 296-298.

Ketting, R.F., Haverkamp, T.H., van Luenen, H.G., and Plasterk, R.H. 1999. Mut-7 of C. elegans, required for transposon silencing and RNA interference, is a homolog of Werner syndrome helicase and RNaseD. Cell 99: 133-141.

Ketting, R.F., Fischer, S.E., Bernstein, E., Sijen, T., Hannon, G.J., and Plasterk, R.H. 2001. Dicer functions in RNA interference and in synthesis of small RNA involved in developmental timing in C. elegans. Genes \& Dev. 15: 2654-2659.

Kim, J.K., Gabel, H.W., Kamath, R.S., Tewari, M., Pasquinelli, A., Rual, J.F., Kennedy, S., Dybbs, M., Bertin, N., Kaplan, J.M., et al. 2005. Functional genomic analysis of RNA interference in C. elegans. Science 308: 1164-1167.

Kuhlmann, M., Borisova, B.E., Kaller, M., Larsson, P., Stach, D., Na, J., Eichinger, L., Lyko, F., Ambros, V., Soderbom, F., et al. 2005. Silencing of retrotransposons in Dictyostelium by DNA methylation and RNAi. Nucleic Acids Res. 33: 6405-6417.

Lee, R.C. and Ambros, V. 2001. An extensive class of small RNAs in Caenorhabditis elegans. Science 294: 862-864.

Lippman, Z. and Martienssen, R. 2004. The role of RNA interference in heterochromatic silencing. Nature 431: 364-370.

Lu, R., Maduro, M., Li, F., Li, H.W., Broitman-Maduro, G., Li, W.X., and Ding, S.W. 2005. Animal virus replication and RNAi-mediated antiviral silencing in Caenorhabditis elegans. Nature 436: 10401043.

Meister, G., Landthaler, M., Patkaniowska, A., Dorsett, Y., Teng, G., and Tuschl, T. 2004. Human Argonaute2 mediates RNA cleavage targeted by miRNAs and siRNAs. Mol. Cell 15: 185-197.

Mello, C.C. and Conte Jr., D. 2004. Revealing the world of RNA interference. Nature 431: 338-342.

Morel, J.B., Godon, C., Mourrain, P., Beclin, C., Boutet, S., Feuerbach, F., Proux, F., and Vaucheret, H. 2002. Fertile hypomorphic ARGONAUTE (agol) mutants impaired in post-transcriptional gene silencing and virus resistance. Plant Cell 14: 629-639.

Mourrain, P., Beclin, C., Elmayan, T., Feuerbach, F., Godon, C., Morel, J.B., Jouette, D., Lacombe, A.M., Nikic, S., Picault, N., et al. 2000. Arabidopsis SGS2 and SGS3 genes are required for posttranscriptional gene silencing and natural virus resistance. Cell 101: 533-542.
Okamura, K., Ishizuka, A., Siomi, H., and Siomi, M.C. 2004. Distinct roles for Argonaute proteins in small RNA-directed RNA cleavage pathways. Genes \& Dev. 18: 1655-1666.

Peragine, A., Yoshikawa, M., Wu, G., Albrecht, H.L., and Poethig, R.S. 2004. SGS3 and SGS2/SDE1/RDR6 are required for juvenile development and the production of trans-acting siRNAs in Arabidopsis. Genes \& Dev. 18: 2368-2379.

Robert, V.J., Sijen, T., van Wolfswinkel, J., and Plasterk, R.H. 2005. Chromatin and RNAi factors protect the C. elegans germline against repetitive sequences. Genes \& Dev. 19: 782-787.

Sijen, T. and Plasterk, R.H. 2003. Transposon silencing in the Caenorhabditis elegans germ line by natural RNAi. Nature 426: 310-314.

Sijen, T., Fleenor, J., Simmer, F., Thijssen, K.L., Parrish, S., Timmons, L., Plasterk, R.H., and Fire, A. 2001. On the role of RNA amplification in dsRNA-triggered gene silencing. Cell 107: 465-476.

Simmer, F., Tijsterman, M., Parrish, S., Koushika, S.P., Nonet, M.L., Fire, A., Ahringer, J., and Plasterk, R.H. 2002. Loss of the putative RNA-directed RNA polymerase RRF-3 makes C. elegans hypersensitive to RNAi. Curr. Biol. 12: 1317-1319.

Smardon, A., Spoerke, J.M., Stacey, S.C., Klein, M.E., Mackin, N., and Maine, E.M. 2000. EGO-1 is related to RNA-directed RNA polymerase and functions in germ-line development and RNA interference in C. elegans. Curr. Biol. 10: 169-178.

Tabara, H., Sarkissian, M., Kelly, W.G., Fleenor, J., Grishok, A., Timmons, L., Fire, A., and Mello, C.C. 1999. The rde-1 gene, RNA interference, and transposon silencing in C. elegans. Cell 99: 123-132.

Tabara, H., Yigit, E., Siomi, H., and Mello, C.C. 2002. The dsRNA binding protein RDE-4 interacts with RDE-1, DCR-1, and a DExH-box helicase to direct RNAi in C. elegans. Cell 109: 861-871.

Tijsterman, M., Ketting, R.F., Okihara, K.L., Sijen, T., and Plasterk, R.H. 2002a. RNA helicase MUT-14-dependent gene silencing triggered in C. elegans by short antisense RNAs. Science 295: 694-697.

Tijsterman, M., Okihara, K.L., Thijssen, K., and Plasterk, R.H. 2002b. PPW-1, a PAZ/PIWI protein required for efficient germline RNAi, is defective in a natural isolate of C. elegans. Curr. Biol. 12: 15351540.

Timmons, L., Court, D.L., and Fire, A. 2001. Ingestion of bacterially expressed dsRNAs can produce specific and potent genetic interference in Caenorhabditis elegans. Gene 263: 103-112.

Tops, B.B., Tabara, H., Sijen, T., Simmer, F., Mello, C.C., Plasterk, R.H., and Ketting, R.F. 2005. RDE-2 interacts with MUT-7 to mediate RNA interference in Caenorhabditis elegans. Nucleic Acids Res. 33: 347-355.

Vastenhouw, N.L., Fischer, S.E., Robert, V.J., Thijssen, K.L., Fraser, A.G., Kamath, R.S., Ahringer, J., and Plasterk, R.H. 2003. A genome-wide screen identifies 27 genes involved in transposon silencing in C. elegans. Curr. Biol. 13: 1311-1316.

Vazquez, F., Vaucheret, H., Rajagopalan, R., Lepers, C., Gasciolli, V., Mallory, A.C., Hilbert, J.L., Bartel, D.P., and Crete, P. 2004. Endogenous trans-acting siRNAs regulate the accumulation of Arabidopsis mRNAs. Mol. Cell 16: 69-79.

Wilkins, C., Dishongh, R., Moore, S.C., Whitt, M.A., Chow, M., and Machaca, K. 2005. RNA interference is an antiviral defence mechanism in Caenorhabditis elegans. Nature 436: 1044-1047.

Williams, R.W. and Rubin, G.M. 2002. ARGONAUTE1 is required for efficient RNA interference in Drosophila embryos. Proc. Natl. Acad. Sci. 99: 6889-6894.

Winston, W.M., Molodowitch, C., and Hunter, C.P. 2002. Systemic RNAi in C. elegans requires the putative transmembrane protein SID-1. Science 295: 2456-2459.

Wood, W.B., ed. 1988. The nematode Caenorhabditis elegans. Cold Spring Harbor Laboratory, Cold Spring Harbor, NY.

Xie, Z., Kasschau, K.D., and Carrington, J.C. 2003. Negative feedback regulation of Dicer-Like1 in Arabidopsis by microRNA-guided mRNA degradation. Curr. Biol. 13: 784-789.

Xie, Z., Johansen, L.K., Gustafson, A.M., Kasschau, K.D., Lellis, A.D., Zilberman, D., Jacobsen, S.E., and Carrington, J.C. 2004. Genetic and functional diversification of small RNA pathways in plants. PLoS Biol. 2: E104. 

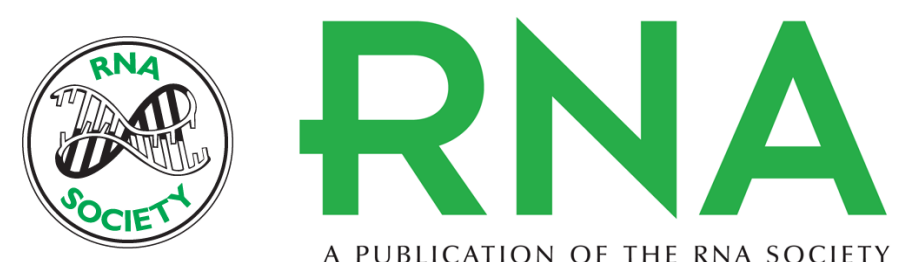

A PUBLICATION OF THE RNA SOCIETY

\section{Interacting endogenous and exogenous RNAi pathways in Caenorhabditis elegans}

ROSALIND C. LEE, CHRISTOPHER M. HAMMELL and VICTOR AMBROS

RNA 2006 12: 589-597

References This article cites 45 articles, 15 of which can be accessed free at: http://rnajournal.cshlp.org/content/12/4/589.full.html\#ref-list-1

License

Email Alerting Receive free email alerts when new articles cite this article - sign up in the box at the Service top right corner of the article or click here.

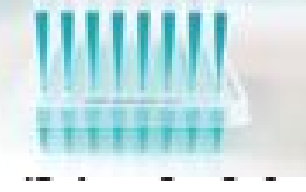

